NBER WORKING PAPER SERIES

\title{
ESTIMATING THE EXPECTED MARGINAL RATE OF SUBSTITUTION: EXPLOITING IDIOSYNCRATIC RISK
}

\author{
Robert P. Flood \\ Andrew K. Rose \\ Working Paper 10805 \\ http://www.nber.org/papers/w10805 \\ NATIONAL BUREAU OF ECONOMIC RESEARCH \\ 1050 Massachusetts Avenue \\ Cambridge, MA 02138 \\ September 2004
}

Flood is Senior Economist, Research Department, International Monetary Fund. Rose is B.T. Rocca Jr. Professor of International Business, Haas School of Business at the University of California, Berkeley, NBER Research Associate, and CEPR Research Fellow. For comments, we thank workshop participants at Dartmouth, SMU, Rich Lyons, and Yangru Wu. Rose thanks INSEAD and SMU for hospitality during the course of this research. The data set, sample output, and a current version of this paper are available at http://faculty.haas.berkeley.edu/arose. The views expressed herein are those of the author(s) and not necessarily those of the National Bureau of Economic Research.

(C2004 by Robert P. Flood and Andrew K. Rose. All rights reserved. Short sections of text, not to exceed two paragraphs, may be quoted without explicit permission provided that full credit, including $\odot$ notice, is given to the source. 
Estimating the Expected Marginal Rate of Substitution: Exploiting Idiosyncratic Risk

Robert P. Flood and Andrew K. Rose

NBER Working Paper No. 10805

September 2004

JEL No. G14

\begin{abstract}
$\underline{\text { ABSTRACT }}$
This paper develops a simple but general methodology to estimate the expected intertemporal marginal rate of substitution or "EMRS", using only data on asset prices and returns. Our empirical strategy is general, and allows the EMRS to vary arbitrarily over time. A novel feature of our technique is that it relies upon exploiting idiosyncratic risk, since theory dictates that idiosyncratic shocks earn the EMRS. We apply our methodology to two different data sets: monthly data from 1994 through 2003, and daily data for 2003. Both data sets include assets from three different markets: the New York Stock Exchange, the NASDAQ, and the Toronto Stock Exchange. For both monthly and daily frequencies, we find plausible estimates of EMRS with considerable precision and time-series volatility. We then use these estimates to test for asset integration, both within and between stock markets. We find that all three markets seem to be internally integrated in the sense that different assets traded on a given market share the same EMRS. The technique is also powerful enough to reject integration between the three stock markets, and between stock and money markets.

Robert P. Flood

Research Department, IMF

$70019^{\text {th }}$ Street, NW

Washington, DC 20431

rflood@imf.org

Andrew K. Rose

Haas School of Business Administration

University of California

Berkeley, CA 94720-1900

and NBER

arose@haas.berkeley.edu
\end{abstract}




\section{Introduction}

In this paper, we develop and apply a simple methodology to estimate the expected intertemporal marginal rate of substitution (hereafter "EMRS"). We do this for two reasons. First, it is of intrinsic interest. Second, when different series for the EMRS are estimated for different markets, comparing these estimates provides a natural yet powerful test for integration between markets. Our method is novel in that it exploits information in asset-idiosyncratic shocks.

While the primary objective of this paper is methodological, we illustrate our technique by applying it to monthly and daily data covering firms from large American and Canadian stock exchanges. Our method delivers plausible EMRS estimates with considerable precision.

Estimates from different markets can be distinguished from each other and from the Treasury bill equivalent.

Section 2 motivates our measurement by providing a number of macroeconomic applications. We then present our methodology; implementation details are discussed in the following section. Our empirical results are presented in section 5, while the paper ends with a brief conclusion.

\section{Why Should Macroeconomists Care About Asset Market Integration?}

We begin with a conventional intertemporal asset pricing condition:

$$
p_{t}^{j}=E_{t}\left(m_{t+1} x_{t+1}^{j}\right)
$$


where: $p_{t}^{j}$ is the price at time t of asset $\mathrm{j}, \mathrm{E}_{\mathrm{t}}()$ is the expectations operator conditional on information available at $\mathrm{t}, m_{t+1}$ is the time-varying intertemporal marginal rate of substitution (MRS), used to discount income accruing in period $\mathrm{t}+1$ (also known as the stochastic discount factor, marginal utility growth, or pricing kernel), and $x_{t+1}^{j}$ is income received at $\mathrm{t}+1$ by owners of asset $\mathrm{j}$ at time $\mathrm{t}$ (the future value of the asset plus any dividends or other income).

We adopt the standard definition of asset integration - two portfolios are said to be integrated when they are priced by the same stochastic discount factor. Here "priced" means that equation (1) holds for the assets in question. Equation (1) involves the moments of $m_{t+1}$ and $x_{t+1}^{j}$, not the realized values of those variables. In particular, for integration we do not required realized values of $m_{t+1}$ to be equated across assets or agents pricing assets.

Although many moments of $m_{t+1}$ are involved in asset market integration, the object of interest to us in this study is $E_{t} m_{t+1}$ the time t expectation of the intertemporal marginal rate of substitution (EMRS). We concentrate on the first moment for three reasons. First, the expectation of the MRS, $E_{t} m_{t+1}$ is intrinsically important; it lies at the heart of much intertemporal macroeconomic and financial economics and is virtually the DNA of modern aggregate economics. Second, it is simple to measure with high statistical accuracy. Third, cross- market differenc es in estimated values of $E_{t} m_{t+1}$ are statistically distinguishable, providing powerful evidence concerning market integration. ${ }^{1}$ We are testing only for first-moment equality when many additional moments are used in asset pricing; thus, ours it a test of a necessary condition for integration. If we reject equality of the first moment, we can reject integration, but failing to reject first-moment equality is consistent with (but does not imply) complete integration. 


\subsection{Motivation}

Asset market integration is a topic of continuing interest in international finance, see e.g., Adam et. al. (2002). It is of special interest in Europe where continuing monetary and institutional integration have lead to lower barriers to asset trade inside the EU. But there are a number of compelling reasons why most policy-oriented macroeconomists should be interested in asset market integration.

When macroeconomic modeling was based on descriptive structure a generation ago, market integration was not very relevant to macro. Modern macroeconomic models, however, are usually built on the assumption that agents maximize an intertemporal utility function in a stochastic setting (e.g., King and Rebelo 2000), and Clarida, Gali and Gertler, 1999). In such macro models our equation (1) could be used to determine bond holdings and might look like:

$$
\frac{1}{\left(1+i_{t}\right)}=E_{t}\left(\frac{\rho u_{c}\left(c_{t+1}\right)^{*} q_{t}}{u_{c}\left(c_{t}\right)^{*} q_{t+1}}\right) .
$$

The corresponding equation to determine stock holding or to value firm revenues would be:

$$
p_{t}=E_{t}\left(\frac{\rho u_{c}\left(c_{t+1}\right) * q_{t}}{u_{c}\left(c_{t}\right) * q_{t+1}} x_{t+1}\right)
$$

In equations (2) and (3), $0<\rho<1$ is a constant; $u\left(c_{t}\right)$ is a concave period flow of utility function with argument period t consumption and $u_{c}\left(c_{t}\right)$ is its partial derivative with respect to period $\mathrm{t}$ 
consumption. The price level at time $\mathrm{t}$ is $q_{t}$; the one-period interest rate is $i_{t} ; x_{t+1}$ and $p_{t}$ are the aggregate counterparts to the symbols defined in equation (1).

Equation (2) is the basis for the modern IS curve; equation (3) is an efficiency condition for investment undertaken in period t. The point is that equations (2) and (3) both use the same stochastic discount rate, $m_{t+1}=\frac{\rho u_{c}\left(c_{t+1}, . .\right)^{*} q_{t}}{u_{c}\left(c_{t}, . .\right)^{*} q_{t+1}}$, to evaluate short-term bonds (equation 2$)$ and the payoffs from real productive assets (equation 3).

In our terminology, modern macro models assume markets pricing bonds and real assets to be integrated - both use the same stochastic discount rate. Asset market integration is a transmission channel of monetary policy in these models. In policy models, monetary authorities adjust $\left(1+i_{t}\right)$ in response to the current and expected future state of the economy, see e.g., Clarida, Gali and Gertler (1999). One transmission mechanism has $m_{t+1}$ adjusting in equation (2) with the discount rate transmitting policy to other decisions, as in equation (3). Cross-market and cross-decision equality of intertemporal discount rates is a substantive assumption. Testing for asset market integration between stocks and bonds (which we do below) can be thought of as a specification test for the modern IS curve.

The IS curve is just one manifestation of asset market integration. Agents in many modern macro models use the same intertemporal discount rate to evaluate all intertemporal decisions - consuming vs. saving (the IS curve), enjoying leisure now vs. enjoying it later (the labor supply schedule), investing savings in various assets, and so on. Because these models typically use a single intertemporal discount rate, asset market integration is an essential ingredient. 
In open economy work, e.g., Obstfeld and Rogoff (2000), capital market integration plays an even more substantive role. It is present in all the ways of closed-economy models, plus it plays a role in cross-currency and other international asset trading possibilities. Integration manifests itself perhaps most clearly in pricing foreign-currency bonds. The foreign- currency bond pricing condition is:

$$
1=E_{t}\left(m_{t+1}^{*} * \frac{s_{t+1}\left(1+i_{t}^{*}\right)}{s_{t}}\right),
$$

where $m_{t+1}^{*}$ is the discount rate used to price foreign currency assets, $s_{t}$ is the domestic-currency price of foreign exchange, and $i_{t}^{*}$ is the foreign-currency interest rate. Another way to write equation (4) is:

$$
1=\operatorname{COV}\left(m_{t+1}^{*}, \frac{s_{t+1}\left(1+i_{t}^{*}\right)}{s_{t}}\right)+E_{t} m_{t+1}^{*} E_{t}\left(\frac{s_{t+1}\left(1+i_{t}^{*}\right)}{s_{t}}\right) .
$$

When pricing of foreign-currency securities is integrated with pricing of domestic-currency securities, $E_{t} m_{t+1}^{*}=E_{t} m_{t+1}=1 /(1+i)$ and equation (5) becomes

$$
1=\operatorname{COV}\left(m_{t+1}, \frac{s_{t+1}\left(1+i_{t}^{*}\right)}{s_{t}}\right)+\frac{1+i_{t}^{*}}{1+i_{t}} E_{t}\left(\frac{s_{t+1}}{s_{t}}\right),
$$

which is familiar from the work of Hodrick (1987). If, however, bond markets are not integrated internationally, equation (5) becomes: 


$$
1=\operatorname{COV}_{t}\left(m_{t+1}^{*}, \frac{s_{t+1}\left(1+i_{t}^{*}\right)}{s_{t}}\right)+\frac{\left(1+i_{t}^{*}\right) E_{t} m_{t+1}^{*}}{\left(1+i_{t}\right) E_{t} m_{t+1}} E_{t}\left(\frac{s_{t+1}}{s_{t}}\right)
$$

Without integration, the size of $\theta_{t}=\frac{E_{t} m_{t+1}^{*}}{E_{t} m_{t+1}}$ is uncertain and varies through time. Tests of uncovered interest parity (UIP) set both $\operatorname{COV}_{t}\left(m_{t+1}^{*}, \frac{s_{t+1}\left(1+i_{t}^{*}\right)}{s_{t}}\right)=0$ and $\theta_{t}=1$, Hodrick (1987).

When such tests reject UIP they are usually interpreted in terms of $\operatorname{COV}\left(m_{t+1}^{*}, \frac{s_{t+1}\left(1+i_{t}^{*}\right)}{s_{t}}\right)$ being non-zero and correlated with interest rate regressors, but this need not be the whole story. For instance, Alvarez, Atkeson, and Kehoe (2002) interpret the UIP puzzle as well as excess volatility results using segmented asset markets, i.e., $\theta_{t} \neq 1$.

There are clearly many reasons why macroeconomists (with either a domestic or international focus) should be interested in asset integration.

\section{Methodology}

Consider a standard decomposition of equation (1):

$$
p_{t}^{j}=E_{t}\left(m_{t+1} x_{t+1}^{j}\right)=\operatorname{COV}_{t}\left(m_{t+1}, x_{t+1}^{j}\right)+E_{t}\left(m_{t+1}\right) E_{t}\left(x_{t+1}^{j}\right)
$$

where $\mathrm{COV}_{t}()$ denotes the conditional covariance operator. It is useful to rewrite this as

$$
x_{t+1}^{j}=-\left[1 / E_{t}\left(m_{t+1}\right)\right] \operatorname{COV} V_{t}\left(m_{t+1}, x_{t+1}^{j}\right)+\left[1 / E_{t}\left(m_{t+1}\right)\right] p_{t}^{j}+\varepsilon_{t+1}^{j} \text {, or }
$$




$$
x_{t+1}^{j}=\delta_{t}\left(p_{t}^{j}-C O V_{t}\left(m_{t+1}, x_{t+1}^{j}\right)\right)+\varepsilon_{t+1}^{j}
$$

where $\varepsilon_{t+1}^{j} \equiv x_{t+1}^{j}-E_{t}\left(x_{t+1}^{j}\right)$, a prediction error orthogonal to information at time t, and

$\delta_{t} \equiv 1 / E_{t}\left(m_{t+1}\right)$. The latter time-series vector is the set of parameters of interest to us. In an integrated market without trading frictions, it is identical for all assets, since the first moment of the marginal rate of substitution should be equal inside integrated financial markets. Our work below is essentially concerned with exploiting and testing this restriction.

\subsection{Two Earlier Approaches to Parametric Estimation of Market Integration}

It is typical in domestic finance to make equation (9) stationary by dividing the equation by $p_{j, t}$, resulting in:

$$
x_{t+1}^{j} / p_{t}^{j}=\delta_{t}\left(1-\operatorname{COV}_{t}\left(m_{t+1}, x_{t+1}^{j} / p_{t}^{j}\right)\right)+\varepsilon_{t+1}^{j},
$$

where $\varepsilon_{t+1}^{j}$ is redefined appropriately. This normalization converts equation (9) into a traditional asset-pricing equation. That is, it breaks one-period asset returns, $x_{t+1}^{j} / p_{t}^{j}$, into the risk-free market return, $\delta_{t} \equiv 1 / E_{t}\left(m_{t+1}\right)$, and asset-specific period risk premia, the covariance term. Equation (10) is given economic content by adding two assumptions:

1) Rational Expectations: $\varepsilon_{t+1}^{j}$ is assumed to be uncorrelated with information available at time $\mathrm{t}$, and 
2) Covariance Model: $\operatorname{COV}_{t}\left(m_{t+1}, x_{t+1}^{j} / p_{t}^{j}\right)=\beta_{0}^{j}+\Sigma_{i} \beta_{i}^{j} f_{i, t}$, for the relevant sample,

where: $\beta_{0}^{j}$ is an asset-specific intercept, $\beta_{i}^{j}$ is a set of I asset-specific factor coefficients and $f_{i, t}$ a vector of time-varying factors. Both assumptions are common in the literature; Campbell, Lo and MacKinlay (1997) and Cochrane (2001) provide excellent discussions. With these two assumptions, equation (10) becomes a panel estimating equation. Time-series variation is used to estimate the asset-specific factor loadings $\{\beta\}$, coefficients that are constant across time. Estimating these factor loadings is a key objective of this research program.

In practice, many empirical asset pricing modelers $\operatorname{set} \delta(t)=1+i(t)$, where $i(t)$ is an appropriate short-term riskless interest rate. ${ }^{2}$ That is, the EMRS is simply equated with e.g., the Treasury-bill rate; it is not estimated at all. While this simplifies empirical work considerably, this assumes integration between stock and money markets, one of the very assumptions we wish to test rather than make.

The first approach to testing asset market integration between a pair of markets makes one of the factors, say the first one, equal to a market ident ifier. This allows cross-sectional estimation of a market-specific effect each period. For a set of risk factors that are held to price assets in both markets, the market-specific effects should all be zero under the null of integration Rejecting the joint null hypothesis - but maintaining rational expectations - rejects either market integration, or the risk pricing model (or both).

Two points are essential to the first approach. First, it is based on the Finance standard where the risk premium is postulated to be a function (usually linear) of a set of aggregate risks. 
Second, the market integration test is tested as part of a joint hypothesis that includes the aggregate risks that model risk premia.

A second approach is provided by Flood and Rose (2003), who follow the spirit of Roll and Ross (1980) in testing for market integration based on cross-market equality of $\delta_{t}$. Flood and Rose differ slightly from the Finance standard and normalize by $p_{t-1}^{j}$ instead of $p_{t}^{j}$

$$
x_{t+1}^{j} / p_{t-1}^{j}=\delta_{t}\left(\left(p_{t}^{j} / p_{t+1}^{j}\right)-\operatorname{COV}_{t}\left(m_{t+1}, x_{t+1}^{j} / p_{t-1}^{j}\right)\right)+\varepsilon_{t+1}^{j}
$$

In this equation, the factor loadings $\{\beta\}$ (from the model used to proxy the covariance model) can still be estimated. But in addition, cross-sectional variation can be used to estimate $\{\delta\}$, the coefficients of interest that represent the EMRS and are time varying but common to all assets. Still, this approach - in common with the traditional approach that relies on (10) - requires correct specification of $\operatorname{COV}_{t}\left(m_{t+1}, x_{t+1}^{j} / p_{t}^{j}\right)$ in the form of the factor model $\beta_{0}^{j}+\Sigma_{i} \beta_{i}^{j} f_{i, t}$. If the latter is mis-specified, the $\{\delta\}$ estimates will also be incorrect.

The traditional finance approach allows one to estimate a covariance model and factor loadings (betas) with precision, at the expense of precluding estimation of the EMRS (since it is assumed to be the T-bill return). The second approach is oriented towards estimating the EMRS, but still requires specification of a covariance model. We now continue further down this road and develop a third approach which is even more geared towards estimating the EMRS; it does not require any explicit covariance model specification.

\subsection{A New Strategy}


In this paper, we rely on a different normalization. Suppose we observe $\tilde{p}_{t}^{j}$, which is defined to be the value of $p_{t}^{j}$ conditional on idiosyncratic information (available at time t) being set to zero. Consider the regression:

$$
\ln \left(p_{t}^{j} / p_{t-1}^{j}\right)=\alpha_{0}^{j}+\sum_{i=1}^{N} \alpha_{i}^{j} f_{t}^{i}+v_{t}^{j},
$$

where the $f_{t}^{i}$ are a set of aggregate factors, e.g., the log of unity plus average price growth, and $v_{t}^{j}$, the residual, is the idiosyncratic part of asset $\mathrm{j}$ price return. From the definition of $\tilde{p}_{t}^{j}$,

$$
\tilde{p}_{t}^{j}=p_{t-1}^{j} \exp \left(\alpha_{0}^{j}+\sum_{i=1}^{N} \alpha_{i}^{j} f_{t}^{i}\right)
$$

which is $p_{t}^{j}$ with its idiosyncratic part set to zero.

Normalizing by $\tilde{p}_{t}^{j}$ delivers:

$$
x_{t+1}^{j} / \tilde{p}_{t}^{j}=\delta_{t}\left[\left(p_{t}^{j} / \tilde{p}_{t}^{j}\right)-C O V_{t}\left(m_{t+1}, x_{t+1}^{j} / \tilde{p}_{t}^{j}\right)\right]+\varepsilon_{t+1}^{j} .
$$

The first term inside the brackets, $\left(p_{t}^{j} / \tilde{p}_{t}^{j}\right)$, equals $\exp \left(v_{t}^{j}\right)$, which is a function of only idiosyncratic information. The second term, $\operatorname{COV}_{t}\left(m_{t+1}, x_{t+1}^{j} / \tilde{p}_{t}^{j}\right)$ is the covariance of the unknown market discount rate, $m_{t+1}$, with the synthetic return, $x_{t+1}^{j} / \tilde{p}_{t}^{j}$ Similar to the risk premium assumption in Finance, we assume $\operatorname{COV}_{t}\left(m_{t+1}, x_{t+1}^{j} / \tilde{p}_{t}^{j}\right)$ moves only because of 
aggregate phenomena. Since idiosyncratic risk, $\left(p_{t}^{j} / \tilde{p}_{t}^{j}\right)$ is orthogonal to systematic risk, $\operatorname{COV}_{t}\left(m_{t+1}, x_{t+1}^{j} / \tilde{p}_{t}^{j}\right),(13)$ can be decomposed as

$$
x_{t+1}^{j} / \tilde{p}_{t}^{j}=\delta_{t}\left(p_{t}^{j} / \tilde{p}_{t}^{j}\right)-\delta_{t} \operatorname{COV}_{t}\left(m_{t+1}, x_{t+1}^{j} / \tilde{p}_{t}^{j}\right)+\varepsilon_{t+1}^{j}=\delta_{t} \exp \left(v_{t}^{j}\right)+u_{t+1}^{j}
$$

where $u_{t+1}^{j} \equiv \varepsilon_{t+1}^{j}-\delta_{t} \operatorname{COV}_{t}\left(m_{t+1}, x_{t+1}^{j} / \tilde{p}_{t}^{j}\right)$. By design, both parts of the composite error term are orthogonal to the only regressor, $\exp \left(v_{t}^{j}\right)=p_{t}^{j} / \tilde{p}_{t}^{j}$. The first part, $\varepsilon_{t+1}^{j}$, is a forecasting error which is unrelated to all information at time t by rational expectations. The second part, $\operatorname{COV}_{t}\left(m_{t+1}, x_{t+1}^{j} / \tilde{p}_{t}^{j}\right)$ is unaffected by any idiosyncratic phenomena. Since both terms are orthogonal to the regressor that represents idiosyncratic risk, $\left(p_{t}^{j} / \tilde{p}_{t}^{j}\right)$, the coefficients of interest, $\{\delta\}$, can be consistently estimated via (14). A correct empirical specification of $\operatorname{COV}_{t}\left(m_{t+1}, x_{t+1}^{j} / \tilde{p}_{t}^{j}\right)$ would lead to more efficient estimation of $\{\delta\}$. However, an empirical specification of $\operatorname{COV}_{t}\left(m_{t+1}, x_{t+1}^{j} / \tilde{p}_{t}^{j}\right)$ is unnecessary for consistent estimation.

The basic idea of this study and the essential way it differs from previous work is that we use asset-idiosyncratic shocks to identify and measure the expected marginal rate of substitution (or rather, its inverse), $\{\delta\}$. This stands typical Finance methodology - the first approach discussed above - on its head. In traditional asset-pricing Finance, idiosyncratic risk is irrelevant and orthogonal to the center-piece measures of aggregate risk. By their nature, idiosyncratic risks are easy to insure against and hence carry no risk premium. While idiosyncratic shocks carry no information about individual asset risk premia, they are loaded with information relevant to market aggregates. Our test for asset market integration is simple; we check if the implied prices of carrying idiosyncratic risks - measures of the expected marginal rate of 
substitution - are equal across portfolios. If equality of the estimated EMRS cannot be rejected, then our test cannot reject cross-portfolio integration. If, however, we can reject equality then we also reject integration.

Our normalization has the advantage - in common with the strategy of Flood-Rose (2003) - that it allows estimation of $\{\delta\}$. However, it does not rely directly on a correctly specified asset pricing model. That is, we do not explicitly rely on a model of $\operatorname{COV}_{t}\left(m_{t+1}, x_{t+1}^{j} / \tilde{p}_{t}^{j}\right)$, (such as, e.g., the CAPM used by Bekaert and Harvey, 1995).

The essential difference between our method and traditional methods is that we substitute a representation of price movements plus an orthogonality condition, for a model of $\operatorname{COV}_{t}\left(m_{t+1}, x_{t+1}^{j}\right)$, which incorporates a similar orthogonality condition. The advantage of our method is that it deals only with observable variables. The stochastic discount rate $m_{t+1}$ is unobservable as are its moments. When we project asset price movements onto a set of aggregate factors, we are taking the same stand on relevant aggregates that others take when they model $\operatorname{COV}_{t}\left(m_{t+1}, x_{t+1}^{j}\right)$. The advantage of our method is that it leaves a highly volatile regressor - idiosyncratic shocks - attached to $\delta_{t}=1 / m_{t+1}$.

Our methodology has a number of other strengths. First, it is based on a general intertemporal theoretical framework, unlike other measures of asset integration such as stock market correlations (see the discussion in e.g., Adam et. al. 2002). Second, we do not need to model the EMRS directly; we allow it to vary over time in a completely general fashion. Third, the technique requires only accessible and reliable data on asset prices and returns. Fourth, the methodology can be used at a full range of frequencies. Fifth, the technique can be used to compare estimates of EMRS across many different classes of intertemporal decisions, including saving decisions that involve domestic and foreign stocks, bonds, and commodities. Sixth, the 
technique is easy to implement and can be applied with standard econometric packages; no specialized software is required. Finally, the technique is focused on estimating an intrinsically interesting object, the (inverse of the) expected marginal rate of substitution.

\section{Empirical Implementation}

In practice $\tilde{p}_{t}^{j}$ is an unobservable variable. Thus, we use an observable statistical counterpart derived from an empirical model, denoted $\hat{p}_{t}^{j}$ (we note that this induces measurement error, an issue we handle below). We do this in a straightforward way, using simple time-series regressions that link individual asset-price returns to the average. In particular, we estimate the following $\mathrm{J}$ time series regressions via ordinary least squares (OLS):

$$
\ln \left(p_{t}^{j} / p_{t-1}^{j}\right)=a_{j}+b_{j} * \ln \left(\bar{p}_{t} / \bar{p}_{t-1}\right)+v_{t}^{j}
$$

where $a_{\mathrm{j}}$ and $b_{\mathrm{j}}$ are fixed regression coefficients, $\bar{p}_{t}$ is the market-wide average price and $v_{t}^{j}$ is the time-t asset idiosyncratic shock. This equation has a natural and intuitive interpretation; it models the first-difference of the natural logarithm of a particular asset price as a linear function of the price growth of the market. Estimates of equation (15) allow us to produce the fitted value of $\hat{p}_{t}^{j}$, which we define as:

$$
\hat{p}_{t}^{j} \equiv p_{t-1}^{j} * \exp \left(\hat{a}_{j}+\hat{b}_{j} \ln \left(\bar{p}_{t} / \bar{p}_{t-1}\right)\right)
$$


We are not particularly attached to this specific model of $\hat{p}_{t}^{j}$. For instance, one could employ the Kalman filter to avoid using future data and allow for moving coefficient estimates. Alternatively one could add additional regressors to equation (15) to control for more aggregate factors. ${ }^{3}$ We have assumed that the log first-difference of prices is linear in the market; one could change the particular functional form assumption. We have used a time-series approach to estimating $\hat{p}_{t}^{j}$ but a cross-sectional approach is also possible. ${ }^{4}$ None of these assumptions are critical; they simply seem to work in practice. ${ }^{5}$ But while this particular setup has delivered sensible results, we stress that one only needs some model for $\hat{p}_{t}^{j}$, not this precise one.

\subsection{Estimation}

We are fundamentally interested in estimating $\{\delta\}$ from the following model:

$$
x_{t+1}^{j} / \hat{p}_{t}^{j}=\delta_{t}\left(p_{t}^{j} / \hat{p}_{t}^{j}\right)+u_{t+1}^{j}
$$

for assets $\mathrm{j}=1, \ldots, \mathrm{J}$, periods $\mathrm{t}=1, \ldots, \mathrm{T}$. We allow $\left\{\boldsymbol{\delta}_{t}\right\}$ to vary arbitrarily period by period.

Using $\hat{p}_{t}^{j}$ in place of the unobservable $\tilde{p}_{t}^{j}$ might induce important measurement error. Hence it is natural to consider estimation of equation (17) with instrumental variables (IV) for consistent estimation of $\left\{\delta_{t}\right\}$. IV is also known to handle the "generated regressor" issue which has long been known to be associated with potentially overstated precision of standard errors; see Shanken (1992) and Cochrane (2001 and website correction). The latter show that this is not typically very important in practice, especially for monthly data. While IV estimation seems natural, estimation via the Generalized Method of Moments (GMM) also allows us to handle both potential econometric issues, while also not requiring independent and identically 
distributed disturbances. Accordingly, we use and compare three different estimation techniques: OLS (for simplicity), IV, and GMM. As instrumental variables for $\left\{p_{t}^{j} / \hat{p}_{t}^{j}\right\}$, we use the set of time-varying market-wide average prices $\left\{\bar{p}_{t}\right\}$.

\subsection{The Data Sets}

We employ two different data sets. The first is a decade of monthly data, spanning 1994M1 through 2003M12, while the second is a year of daily data for 2003. We use different frequencies both for intrinsic interest and to check the sensitivity of our techniques. Though these frequencies are standard in Finance, there is nothing special about them, and there is no obvious reason why our methodology could not be used at either higher or lower frequencies. We focus on stock markets, but again see no reason why bond and other markets could not be considered.

Our American stock data were extracted from the CRSP data base and consist of monthend prices and returns (including dividends, if any) for all firms in the S\&P 500 (as of the end of 2003). We have adjusted for stock splits, and checked and corrected the data for errors. We only retain the 435 companies that have data for the full sample span (this selection does not induce any bias that we can imagine). Since we are interested in estimating and comparing implied EMRS across markets, we also include data from two other markets. First, we add comparable data for the NASDAQ-100 firms, also taken from CRSP. Most S\&P 500 firms are traded on the New York Stock Exchange (NYSE), and we avoid overlap (when we compare the NYSE with the NASDAQ) by simply deleting from the S\&P sample those firms traded on the NASDAQ. Second, we add comparable data for the firms in the S\&P/TSX Composite Index of 
the Toronto Stock Exchange (TSE). This data set is extracted from Datastream, and we convert Canadian dollars into American using comparably timed exchange rates.

For the monthly data set, we have 120 monthly observations on 389 firms from the S\&P 500 traded on the NYSE, 65 different firms from the NASDAQ, and 152 firms from the TSE. For the daily data set, we have data for 247 business days when both the Canadian and American stock exchanges were open, on 440 NYSE firms, 99 NASDAQ firms, and 223 TSE firms.

It has been traditional since at least Fama and MacBeth (1973) to use yields on shorthorizon treasury bills to proxy the risk-free rate, and it is natural for us to compare our estimates of the expected risk-free rate with T-bill returns. We use data on T-bill returns downloaded from the Federal Reserve's website. ${ }^{6}$

Finally, for purposes of estimation, we group our stocks into portfolios, typically twenty. We do this randomly (alphabetical order of ticker), though we see no reason why one could not group firms on the basis of e.g., beta, size, or whatnot. We use portfolios partly to remain within the Finance tradition followed since Fama and MacBeth (1973). But using portfolios also makes our task more difficult, since portfolios have lower idiosyncratic risk and reduce cross-sectional dimensionality. It turns out not to destroy our ability to estimate the parameters of interest to us, though there is no obvious reason why individual securities could not be used in place of portfolios.

\section{Results}

The focus of this paper is estimating the expected marginal rate of substitution We begin with an illustration that relies on monthly data from 400 firms in the S\&P 500, grouped into 
portfolios of twenty firms. ${ }^{7}$ We have 118 observations between February 1994 and November 2003, since we lose an observation at either end of the sample, due to leads/lags.

The three graphs on the left of Figure 1 portray estimates of the EMRS from (17), denoted $\left\{\hat{\delta_{t}}\right\}$. These were estimated using three different techniques: IV, GMM, and OLS, using only the first ten portfolios. The mean of $\left\{\hat{\delta_{t}}\right\}$ is plotted, along with a $+/-2$ standard error confidence interval band. The OLS and GMM point estimates are identical (by construction), and are extremely highly correlated with the IV estimates. The primary differences between the different estimates lie in the standard errors; all three estimators deliver small standard errors, with the GMM standard errors being slightly smaller than those of either IV or OLS (but with more period to period volatility). ${ }^{8}$ Indeed, we almost never find significant differences between the three estimators below, and thus tend to rely on IV below. ${ }^{9}$

Even though we estimate the expected MRS from only ten portfolios, the results seem quite sensible. Most of the estimates of the (inverse of the) expected monthly MRS are just over unity. The sample average of $\left\{\hat{\delta_{t}}\right\}$ over the 118 periods is around 1.0085 , implying an annual MRS of slightly over $1.1\left(=1.0085^{12}\right)$. While somewhat high compared to e.g., Treasury bill returns, this figure is certainly plausible in magnitude. Further, the measures of EMRS are estimated with precision; the confidence interval is barely distinguishable from the means in the plots. Still, the most striking feature of the expected MRS is not its mean, but its volatility over time. The standard deviation of $\left\{\hat{\delta_{t}}\right\}$ is around .04 for all estimators, and the point estimates vary over the decade between .88 and 1.09. This considerable volatility in the expected EMRS mirrors our (2003) results as well as the famously high lower bound of Hansen-Jagannathan $(1991) .^{10}$ 


\subsection{Integration within the S\&P 500}

Do our results depend sensitively on the exact choice of portfolios chosen? An easy way to check is to estimate $\left\{\hat{\delta_{t}}\right\}$ using data from all twenty portfolios and look at the differences from the ten-portfolio estimates. This is done on the right side of Figure 1, which graphs the mean and confidence intervals of the expected MRS for the three estimators. In particular, the graphs on the right portray the difference between $\left\{\hat{\delta_{t}}\right\}$ estimated from all twenty portfolios, and $\left\{\hat{\delta_{t}}\right\}$ estimated from only the last ten portfolios. The differences are economically small; they average around .003 (for all three estimators). They also have large standard errors (of around .011), so that the differences do not appear to be statistically significant. In an integrated market, all securities should deliver the same expected marginal rate of substitution. Figure 1 thus delivers little evidence of significant departures from integration inside the S\&P 500.

The rows on the right of Figure 1 compare $\left\{\hat{\delta_{t}}\right\}$ on a period by period basis for a given estimator. That is, the figures implicitly ask whether the expected MRS for, say, February 1994, is the same when estimated from all twenty portfolios and only from the last ten. This is interesting because equality of $\left\{\hat{\delta_{t}}\right\}$ derived from different assets is a necessary (but not sufficient) condition for market integration. But it is also interesting to compare the entire set of estimated EMRS simultaneously; that is, to test formally for joint equality. If the disturbances $\left\{\hat{u}_{t}^{j}\right\}$ - were normally distributed, this test would be easy to compute via a standard F-test. However and unsurprisingly, there is massive evidence of non-normality in the form of fat tails (leptokurtosis). ${ }^{11}$ Accordingly, we estimate the distribution for our critical values with a conventional bootstrap. With our bootstrapped results, we find the hypothesis of joint equality $\left\{\hat{\delta_{t}}\right\}$ for all 118 observations cannot be rejected at any conventional significance level(for any 
estimator). That is, we cannot reject integration within the S\&P 500. While this might only indicate a lack of statistical power in our techniques, we show later on that it is easy to reject equality of $\left\{\hat{\delta_{t}}\right\}$ across substantively different markets.

\subsection{Estimates of the Expected Marginal Rate of Substitution and Treasury Bills}

The hypothesis of equality of $\left\{\hat{\delta_{t}}\right\}$ cannot be rejected when the twenty stock portfolios are split up. But are the estimated EMRS similar to treasury-bill returns? No. It is easy to generate the risk-free rate using an actual interest rate; we simply create $\vec{\delta}_{t} \equiv\left(1+i_{t}\right)$ where $i_{t}$ is the monthly return on nominal treasury bills. The sample average of $\left\{\vec{\delta}_{t}\right\}$ is around 1.003 (around $3.7 \%$ annualized), somewhat lower than but close to the sample average of $\left\{\hat{\delta_{t}}\right\}$.

But while the first moments of our estimated risk-free rate and the T-bill equivalent are similar, the second moments are not. The T-bill rate has considerably lower time-series volatility than our estimated EMRS. The standard deviation of $\left\{\vec{\delta}_{t}\right\}$ (across time) is .001, which is smaller than that of $\left\{\hat{\delta_{t}}\right\}$ by a factor of over thirty! Since the estimated risk-free rate is so much more volatile than the T-bill equivalent, it is unsurprising that the hypothesis of equality between the two can formally be rejected at any reasonable level of significance. ${ }^{12}$

To summarize, our estimates of the time- varying expectation of marginal rate of substitution are intuitively plausible in magnitude, and precisely estimated. They also display considerable volatility over time. While this variation is consistent with the literature, it is grossly at odds with the smooth T-bill return. Unsurprisingly, we can reject equality between our estimates of EMRS and those of the T-bill. 


\subsection{Other Markets}

What of different markets? Figure 2 provides estimates of the expected marginal rate of substitution (along with a $+/-2$ standard error confidence interval) derived from three different markets: the NYSE, the NASDAQ, and the TSE. In each case, we use twenty portfolios to estimate the expected risk-free rate. The number of available stocks differs by exchange; we use portfolios of nineteen stocks each from the NYSE, but our NASDAQ portfolios contain only

three stocks, and those from the TSE have seven stocks. We estimate $\left\{\hat{\delta_{t}}\right\}$ in the same way as above, using instrumental variables for 118 observations between 1994M2 and 2003M11. To facilitate comparison, we also graph the EMRS implicit in the short Treasury bill return.

For all three markets, the average value of EMRS seems reasonable, being slightly over unity. These are again estimated with considerable precision, even for the NASDAQ; the confidence interval can hardly be distinguished from the mean. But again, the single most striking feature of the estimates is their considerable time-series volatility. The standard deviation (over time) of $\left\{\hat{\delta_{t}}\right\}$ is .04 for both the NYSE and the TSE, and .07 for the NASDAQ. While this is consistent with received wisdom in Finance (e.g., Hansen and Jagannathan, 1991), it contrasts starkly with the smooth T-bill return portrayed at the bottom of Figure 2.

Our results for the three markets are tolerable and consistent with our earlier findings. Different estimators (OLS, GMM, and IV) deliver economically similar results which are statistically close (Hausman tests sometimes reject equality and sometimes do not). There is considerable leptokurtosis. And bootstrapped tests for internal integration indicate no evidence that using e.g., ten NASDAQ portfolios delivers significantly different estimates of the expected MRS from using all twenty NASDAQ portfolios. That is, we find no evidence against internal integration for the three markets. 
We consider these results to be reassuring, given the depth and liquidity of the three advanced stock markets we consider. But they might simply indicate a lack of power in our statistical techniques; after all, they are simply not rejecting a necessary (but not sufficient) test for market integration. Accordingly, as a more stringent test, we also now test formally for integration across markets. This is also a subject of considerable intrinsic interest.

We begin comparing the estimated risk-free rate across markets with a series of scatterplots in Figure 3. The top two graphs on the left of the Figure compare monthly estimates of $\left\{\hat{\delta_{t}}\right\}$ from the NASDAQ and the TSE (on the y-axis) against those derived from the NYSE (on the $\mathrm{x}$-axis). At the bottom-left, we also provide a comparable graph using the T-bill rate on the ordinate. Clearly, the estimates of the expected MRS from the NASDAQ and TSE are correlated with that from the NYSE; the correlation coefficients are .67 and .73 respectively. However they are not identical; the mean absolute difference between the $\left\{\hat{\delta_{t}}\right\}$ derived from the NYSE and the NASDAQ is .04, and almost $10 \%$ are greater than .1 (the analogous figures for the TSE are .02 and $3 \%$ respectively).

It is straightforward to formally test the hypothesis that the estimated EMRS are equal across markets. One way to do this is to test for equality between the estimates graphed in Figure 3. While this is perfectly acceptable (and the method we use for the daily results below), we note that the portfolios used to estimate $\left\{\hat{\delta_{t}}\right\}$ graphed in Figure 3 have different numbers of stocks. Thus they have different degrees of estimation precision. To "balance the playing field" we construct twenty portfolios NYSE with nineteen stocks each. We can then use simple Chow tests to test for equality between $\left\{\hat{\delta_{t}}\right\}$ derived from the twenty NYSE portfolios with those which also use either the three NASDAQ portfolios (of nineteen stocks each), or the eight TSE portfolios (again, of nineteen stocks). When we do so, we find strong evidence against 
integration. The F-test for integration between the NYSE and the NASDAQ is over 13, strongly rejecting the null hypothesis of integration (even allowing for non-normally distributed disturbances that we explore through the bootstrap). The analogous F-test for integration between the NYSE and the TSE is over 8, again inconsistent with the null hypothesis of equality at all significance levels.

Succinctly, while our estimates of the expected MRS are similar for the three different markets, they are significantly different in both the economic and statistical senses. That is, we are able to reject the hypothesis of equal EMRS across markets, and thus market integration. This result is intrinsically interesting, since there are few obvious reasons for this market segmentation. Moreover, they indicate that our methodology is not lacking in statistical power.

\subsection{Daily Results}

Thus far, we have used a decade of monthly data. We now present results derived from the most recent available year of daily data, 2003. We use closing rates for the 245 days when both markets were open, converting Canadian dollar quotes from the TSE into American dollars using a comparable exchange rate. We consider the same three markets, noting in passing that both the American and Canadian markets close at 4:00pm daily in the same time zone.

Figure 4 is the daily analogue to the monthly estimates displayed in Figure 2. In particular, we plot the mean of the expected MRS for all three markets, along with a $+/-2$ standard error confidence interval (the T-bill equivalent is also plotted at the bottom of the figure). We use IV as our estimator, though essentially nothing changes if we use OLS or GMM.

In each case, we present estimate $\left\{\hat{\delta_{t}}\right\}$ using twenty portfolios; each NYSE portfolio has 22 stocks, each NASDAQ portfolio five, and each TSE portfolio eleven. ${ }^{13}$ 
As with the monthly data set, the means of the series again seem reasonable; they are 1.001, 1.002 and 1.001 for the NYSE, NASDAQ and TSE respectively. These magnitudes seem intuitively reasonable, if somewhat high; they are roughly comparable in order of magnitude to the T-bill interest rate, which averaged just over $1 \%$ in 2003 . The series of EMRS are also estimated with considerable precision manifest in tight confidence intervals. There is again evidence of leptokurtosis. Still, the most striking feature of all three series of the estimated MRS is their volatility over time. This is especially true when one compares them with the virtually flat T-bill return. It is little surprise then that the hypothesis that the daily estimates of $\left\{\hat{\delta_{t}}\right\}$ derived from S\&P 500 stock prices are statistically far from the T-bill equivalent $\left\{\vec{\delta}_{t}\right\} .^{14}$

When we check for internal integration within a market (such as S\&P 500 stocks traded on the NYSE) by comparing estimates of $\left\{\hat{\delta_{t}}\right\}$ derived from different sets of portfolios, we are unable to reject the hypothesis of equality at any reasonable confidence interval. That is, we (unsurprisingly) find no evidence against integration within markets.

However, as with the monthly data, integration across markets is another story. The scatter-plots of the estimated daily EMRS at the right side of Figure 3 are analogous to those with monthly data immediately to the left. Both the NASDAQ and the TSE deliver $\left\{\hat{\delta_{t}}\right\}$ that are positively correlated with those from the NYSE; the correlation coefficients are .86 and .69 respectively. The mean absolute differences between the series are around .006 (for both the NASDAQ and the TSE compared to the NYSE), and range to over .02. While these may seem small, they are economically large since they are at a daily frequency. In any case, the series are statistically distinguishable. When we test for equality between the estimates of $\left\{\hat{\delta_{t}}\right\}$ portrayed in Figures 3/4, we find the hypothesis rejected for both the NYSE against the NASDAQ (the F- 
test statistic is over 12) and the NYSE against the TSE (the test statistic is over 17).

Bootstrapping the critical values does not reverse these conclusions.

In brief, our daily data set produces similar results to those of our monthly data set. The estimates of the expected MRS seem intuitively reasonable, and display volatility consistent with that in the literature, but far in excess of the T-bill. While we can never reject the hypothesis of internal integration, we always reject the hypothesis of integration across markets in the sense of equal EMRS.

\section{Conclusion}

In this paper, we have developed a methodology for estimating the expected intertemporal marginal rate of substitution. Our technique relies on exploiting the general fact that idiosyncratic risk, which does not alter any risk premia, should deliver a return equal to the market's expectation of the marginal rate of substitution. This enables us to estimate the expected risk-free rate from equity price data, an object that is intrinsically interesting. Comparing the rates estimated from different markets also provides a natural test for market integration, since integrated markets should share a common expected MRS.

We apply our methodology to a decade of monthly data and a year of daily data, including data on stocks traded on the New York Stock Exchange, the NASDAQ, and the Toronto Stock Exchange. For both data sets, we find intuitive estimates of the expected marginal rate of substitution with reasonable means and considerable volatility over time. We cannot reject the hypothesis that markets are internally integrated in the sense that different portfolios traded on a given market seem to have the same expected marginal rate of substitution. 
However, we find it easy to reject the hypothesis of equal EMRS across markets. This is both of direct interest, and indicates that our technique has considerable statistical power.

There are many possible ways to extend our work. One could add a covariance model e.g., the well-known factor model first developed by Fama and French (1996) - to equation (14). A well-specified covariance model should result in more efficient estimates of the EMRS. Alternatively, one could sort stocks into portfolios in some systematic way (e.g., size, industry, or beta). More factors could be added to the first stage regression, equation (15). More generally, while our use of the $\tilde{p}_{t}^{j}$ normalization has advantages, others might be used instead. Most importantly, while we have been able to reject the hypothesis of integration in the sense of equal expected marginal rates of substitution across markets, we have not explained the reasons for this finding of apparent market segmentation. If our result stands up to scrutiny, this important task remains. 


\section{References}

Adam, Klaus, Tullio Jappelli, Annamaria Menichini, Mario Padula, and Marco Pagano (2002) "Analyse, Compare, and Apply Alternative Indicators and Monitoring Methodologies to Measure the Evolution of Capital Market Integration in the European Union" University of Salerno manuscript.

Alvarez, Fernando, Andrew Atkeson, and Patrick Kehoe (2002) "Money, Interest Rates, and Exchange Rates in Endogenously Segmented Markets" Journal of Political Economy 110-1, 73112.

Bekaert, Geert and Campbell R. Harvey (1995) “Time-Varying World Market Integration” The Journal of Finance 50-1, 403-444.

Campbell, John Y., Andrew W. Lo, and A. Craig MacKinlay (1997) The Econometrics of Financial Markets (Princeton: University Press).

Clarida, Richard, Jordi Gali, and Mark Gertler (1999) “The Science of Monetary Policy: A New Keynesian Perspective” Journal of Economic Literature 37-2, 1661-1707.

Cochrane, John H. (2001) Asset Pricing, Princeton University Press.

Fama, Eugene F. and Kenneth R. French (1996) "Multifactor Explanations of Asset Pricing Anomalies" Journal of Finance 51-1, 55-84.

Fama, Eugene F. and James D. MacBeth (1973) "Risk, Return and Equilibrium: Empirical Tests” Journal of Political Economy 81-3, 607-636.

Flood, Robert P. and Andrew K. Rose (2003) "Financial Integration: A New Methodology and an Illustration” NBER Working Paper \#9880.

Hansen, Lars Peter and Ravi Jagannathan (1991) "Implications of Security Market Data for Models of Dynamic Economies” Journal of Political Economy 99-2, 225-262.

Hodrick, Robert (1987) The Empirical Evidence on the Efficiency of Forward and Futures Foreign Exchange Markets (Harwood, New York).

King, Robert and Sergio Rebelo (2000) "Resuscitating Real Business Cycles" in Handbook of Macroeconomics (John Taylor and Michael Woodford eds.), North-Holland, 927-1007.

Obstfeld, Maurice and Kenneth Rogoff (2000) "New Directions for Stochastic Open Economy Models” Journal of International Economics 50, 117-153.

Roll, Richard, and Stephen A. Ross (1980) “An Empirical Investigation of the Arbitrage Pricing Theory" Journal of Finance 35-5, 1073-1103. 
Shanken, Jay (1992) "On the Estimation of Beta-Pricing Models" Review of Financial Studies 51, 1-33. 


\section{Expected MRS, first 10 portfolios; S\&P 500 1994M2-2003M11 Deltas, with +/- 2 S.E. Confidence Interval}
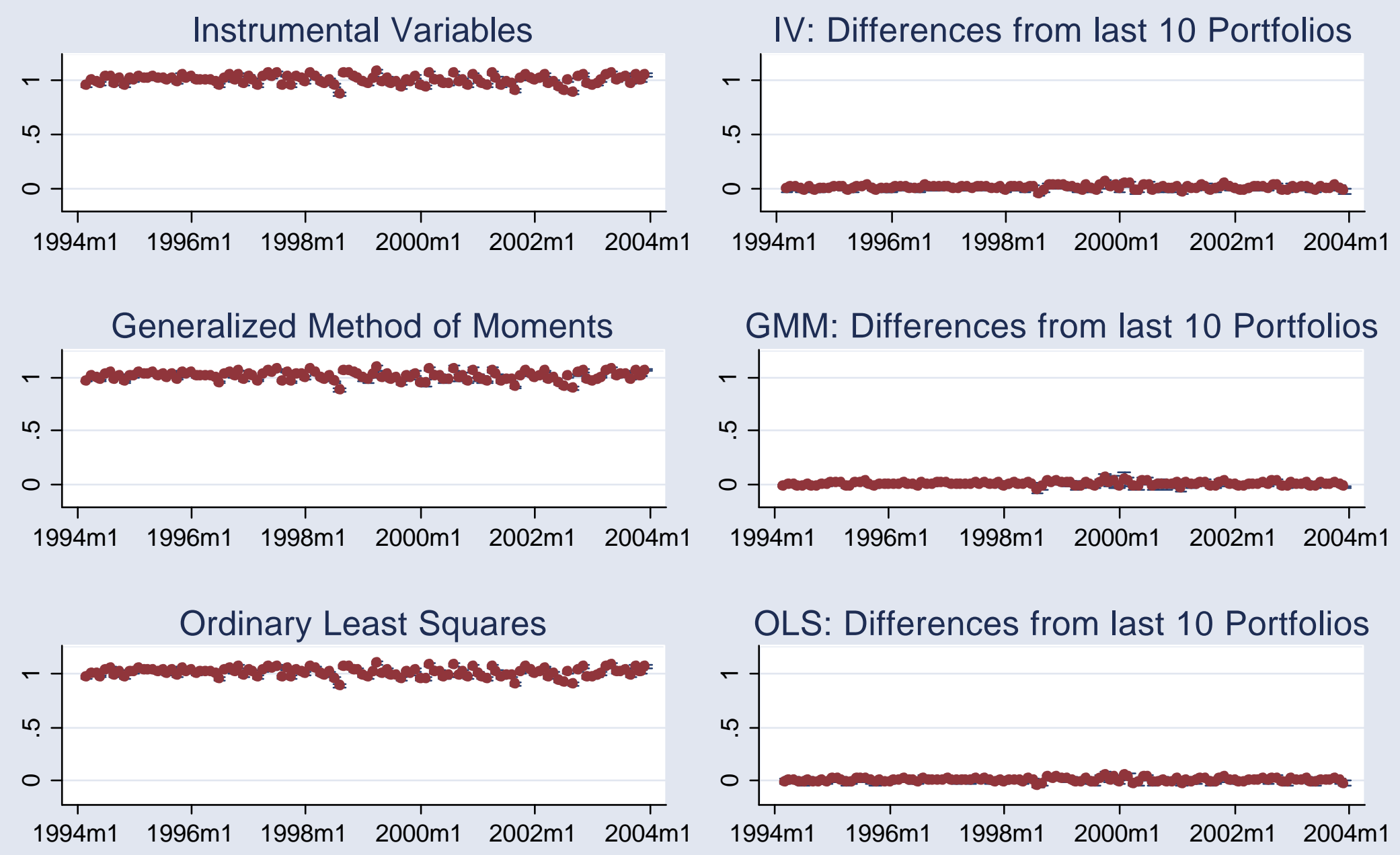

Figure 1: Estimated Expected MRS, Portfolios of 20 S\&P500 firms, 1994M2-2003M11: Different Estimators 


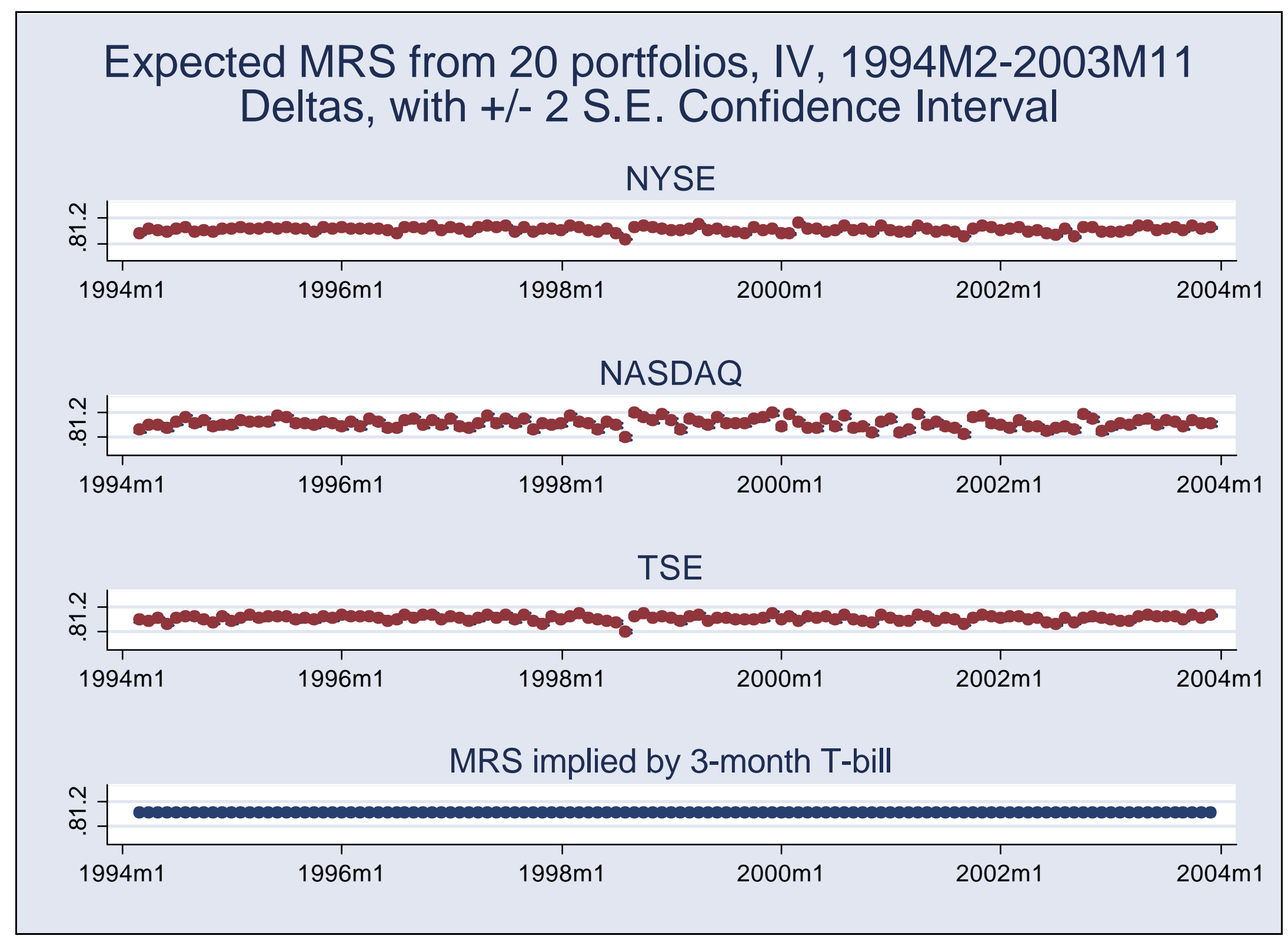

Figure 2: Estimates of Expected Marginal Rate of Substitution, 1994M2-2003M11: Different Markets 


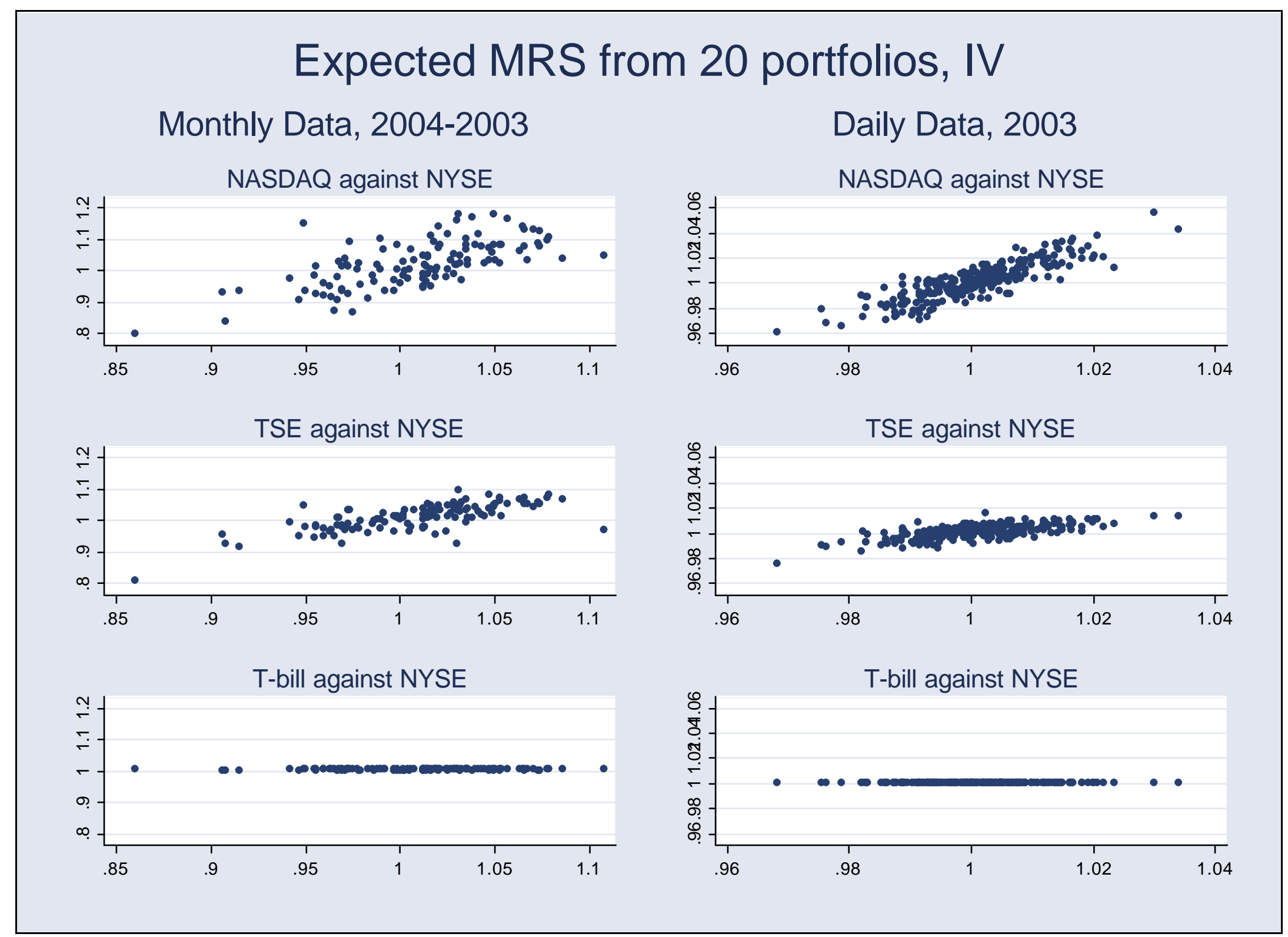

Figure 3: Scatter-plots of Estimated Expected MRS across Markets 


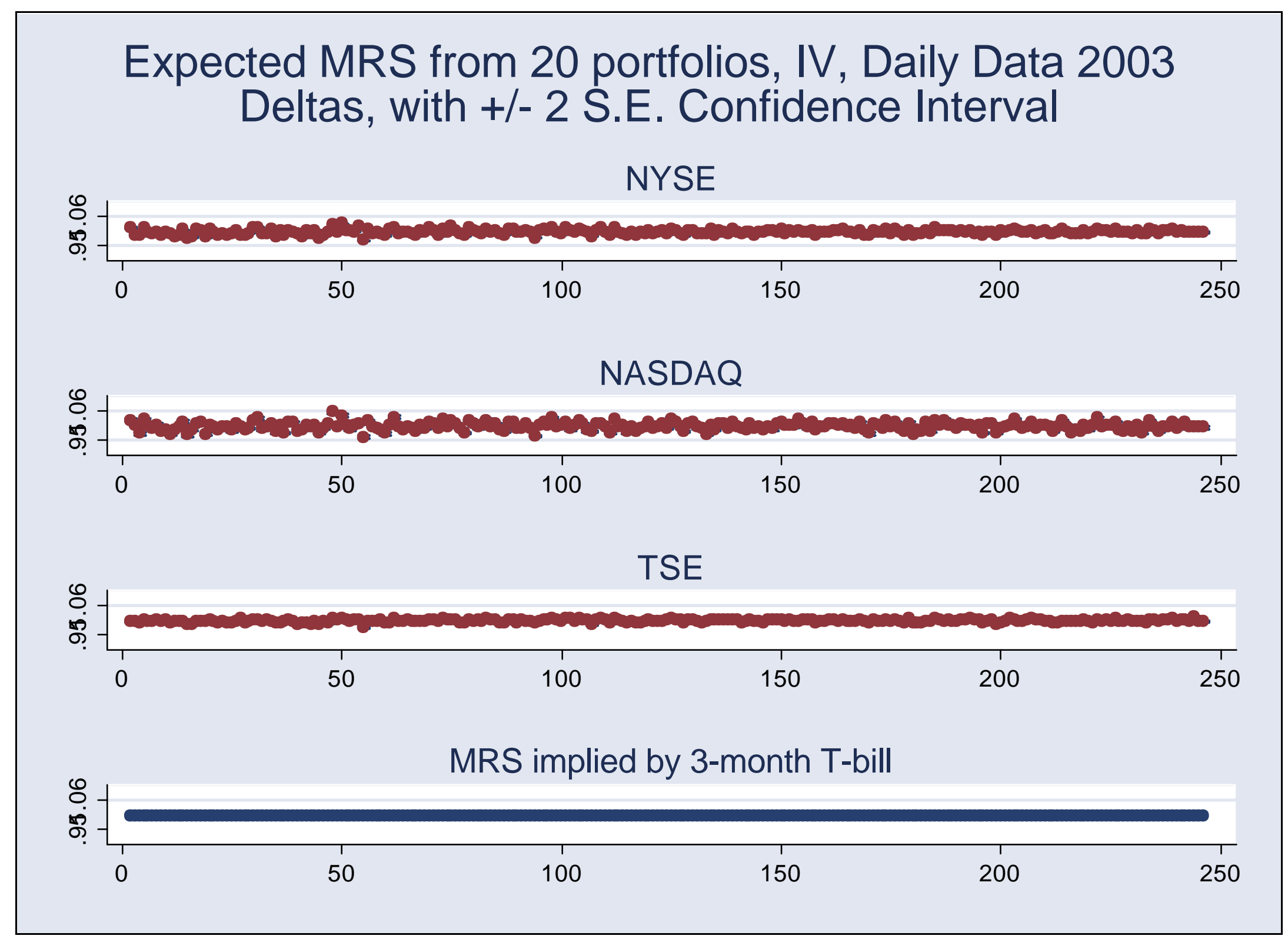

Figure 4: Daily Estimates of Expected Marginal Rate of Substitution, 2003 


\section{Endnotes}

1 In general, there is no guarantee that $m_{t+1}$ is a unique variable; agents behaving according to equation (1) use the entire perceived distribution of $m_{t+1}$ to price assets at $\mathrm{t}$.

2 Alternatively, one can set $\beta_{0}^{j}=0$ for all $\mathrm{j}$.

${ }^{3}$ We have experimented with additional regressors suggested by Fama and French (1996), and they seem to make no difference to our results in practice.

4 Indeed, we have experimented with a cross-sectional analogue to (15), and it delivers economically and statistically similar estimates of $\{\delta\}$.

5 For instance, the median $\mathrm{R}^{2}$ from our twenty estimates of equation (15) is a respectable .77, and the lowest of the twenty $\mathrm{R}^{2} \mathrm{~s}$ is still .59.

${ }^{6}$ In particular, we use closing secondary bid prices on three-month treasury bills for both daily data sets.

7 These firms are traded mostly on the NYSE, but somewhat on the NASDAQ.

8 One can compare the differences between the estimators with a Hausman test. In this case, the difference between the OLS and IV estimators turn s out to be economically small but marginally statistically significant; the hypothesis of equality is rejected at the .006 confidence interval. Also, robust standard errors (either clustered by portfolio or not) are typically even smaller.

9 The exception is our bootstrapped tests for integration, where we tend to use OLS for computational simplicity.

10 The estimates of EMRS have essentially no persistence and are also uncorrelated with traditional finance factors, such as the three used by Fama and French (1996). Adding either an intercept or portfolio-specific intercepts to the estimating equation (17) changes results little, which is unsurprising since the former are small and of marginal significance; the same is basically true of time-specific intercepts. Finally, we have added the three time-varying Fama-French factors to the first stage equation (15); this makes any substantial difference to our results.

11 This is a well-known phenomena; see, e.g., Campbell, Lo, and MacKinlay (1997).

12 The F-test statistic for equality between the expected MRS and the T-bill return is over 50; under the null hypothesis of market integration, it has degrees of freedom $(118,2360)$. Bootstrapping the critical values has no substantive effect on conclusions.

13 The exception is the last NASDAQ portfolio which only has four stocks.

${ }^{14}$ The F-test statistic for equality between the expected MRS and the t-bill return is over 150. 